\title{
Designing Human-Robot Interaction for Dependent Elderlies: a Living Lab Approach
}

\author{
Karine Lan Hing Ting \\ ROSAS/ICD, UMR 6281 \\ Troyes University of Technology \\ 12 rue Marie Curie, Troyes \\ karine.lan@utt.fr
}

\author{
Mustapha Derras \\ Berger Levrault \\ 892 rue Yves Kermen \\ 92100 Boulogne-Billancourt \\ mustapha.derras@berger-levrault.com
}

\author{
Dimitri Voilmy \\ ROSAS/ICD, UMR 6281 \\ Troyes University of Technology \\ 12 rue Marie Curie, Troyes \\ dimitri.voilmy@utt.fr
}

\begin{abstract}
This paper describes the design and evaluation process of a mobile social robotic solution for elderlies, following a living lab approach. The living lab approach combines the principles of human-centred approach and participatory design. The research question at the heart of this study is whether a proper understanding of needs and participation of stakeholders in the design process ensures usefulness and acceptance of the solution. Informed by fieldwork in a retirement home, a prototype of human-robot interaction has been iteratively designed and evaluated with the participation of users. This HRI design serves as the basis to examine the practical acceptance this social robot interaction, as a first step to a broader reflection about the social acceptability of social robots. The first insights of this study are presented in this paper.
\end{abstract}

Mobile social robot, Retirement homes, Human-centred interaction design, Needs analysis, Informing design

\section{INTRODUCTION}

In the current context of the ageing population (according to the UN estimation, in 2050 one out of every five people will be over 60 years old), the challenge is to design new and innovative models to help elderly people achieve healthy ageing and maintain their autonomy. Technological solutions can constitute useful help to independent living. Ambient Assisted Living technologies are introduced in the home to support elderly people, whether for self-care, fall detection/prevention, physiological parameters monitoring, or social interaction services.

A clear interest of research programmes and studies appears on the home: from simple applications or GPS, to "intelligent"/"smart"/future homes - a research area to which the authors of this paper are themselves contributing. Social robots being hypothesized to aid the elderly to live in their homes autonomously for longer and therefore to decrease the burden on our social and healthcare systems, the most recent and interesting studies have focused on their acceptance at home, e.g. [7]. Fewer studies have focused on social robotics use in care institutions and how they could enhance the social life of older people in a retirement home. Those who have, however, and despite their limits [6], show promising results as regards the perception of elderlies living collectively in retirement homes.
Many studies report positive findings with regards to social ties between elderlies (measured by the frequency of contact between elderlies) as well as between elderly and family. Typically, the companion is the topic of conversation. With regards to the perception of the companion robot, narrative records present in a large portion of these studies show that most elderly actually report liking the robots (or their controls, such as a pet toy, for that matter). More generally (both home and institutions), most studies reported positive effects of companiontype robots on both socio-psychological parameters - mood, loneliness, and social connections and communication - and physiological parameters, e.g. stress reduction [3]. However, the risk of social robots increasing social isolation at home is a real issue which still needs to be empirically investigated.

This paper presents the design process - for a new interface and human-robot interaction - and discusses the approach and final objective of this research, of which this paper presents the preliminary study. The reality in this retirement home where our study is being done, is the difficult adoption of everyday technological devices tablets, smartphones, computers - for dependent elderlies living in institutions. The research hypothesis was therefore to trigger elderlies' interaction and use of media content - by / through / with - a tool that would appear as both strange 
(machine) and familiar (human-like form): a mobile social robot.

The reason is that the acceptance of social robots is presumed to differ from the acceptance of other technical innovations, because these robots are not always perceived by their users as being technologies [7]. Also, people who perceive higher levels of anthropomorphism tend to be more positive in evaluating a social robot, perceive higher enjoyment when engaging with it and are more likely to see the robot as a companion. People who enjoy the use of a robot, also think that robot is more easy to use [7]. Based on knowledge from previous studies, and the situation in that home, the research questions guiding this preliminary study are:

$R Q 1$ : what is efficient human-robot interaction, in the case of elderlies and a mobile social robot?

RQ2: how does efficient human-robot interaction contribute to acceptance of social robots?

RQ3: is acceptance of social robots higher if design follows a human-centred and participatory approach?

\section{MOBILE SOCIAL ROBOTICS}

\subsection{Social robots - possible usefulness}

Social robots are characterized by understanding and communicating in a humanlike way, allowing them to behave as social actors and be understood as such by their users [7]. Concerning elderly care, the functionalities of Socially Assistive Robotics are related to the support of independent living by supporting basic activities (eating, bathing, toileting and getting dressed) and mobility (including navigation), providing household maintenance, monitoring of those who need continuous attention and maintaining safety [6]. There is therefore this dual use and perception: (i) social robots can be perceived as utilitarian systems; they are able to perform tasks such as housekeeping, (ii) social robots are recognized as hedonic systems; they offer sociable interaction opportunities to be able to build long-term relationships with their users [9].

\subsection{Social robots in elderly care: previous work}

Studies of social assistive robotics in elderly care [6], [5], [12], range from "service type robots" providing functional assistance, to "companion type robots" providing affective assistance, with sometimes crosschecks between the two categories [6]. Most studies reported positive effects of companion-type robots on both socio-psychological parameters mood, loneliness, and social connections and communication - and physiological parameters, e.g. stress reduction [3]. As regards elderlies living in retirement homes, many studies report positive findings with regards to social ties between elderlies (measured by the frequency of contact between elderlies) as well as between elderly and family. Typically, the companion is the topic of conversation. With regards to the perception of the companion robot, narrative records present in a large portion of these studies show that most elderly actually report liking the robots (or their controls, such as a pet toy, for that matter). However, several limits (methodological and cultural - Japan-only), prevent generalizability of insights in other contexts.

In the Nursebot project, a mobile nursing-assistant robot was developed to assist elderly individuals with mild cognitive and physical impairments, as well as support nurses in their daily activities. The 2 main services were: the task of reminding people of events, guiding them through their environment. The main research focus being navigation, users' perception and acceptance were very briefly tackled, [10] reporting only that "Post-experimental debriefings illustrated a uniform high level of excitement on the side of the elderly."

\subsection{From human-friendly to human-centred}

The approach associated with the terms "humanfriendly robots" [14], "human-friendly robot design" or "human-centred robotics" [14] are more technologyrather than human- centred. Developing humanfriendly robots [8] rests on two key components: (i) Smart interfaces and (ii) Safe mechanisms - to ensure that people are never harmed. From previous experience [13], the authors are convinced of the possibility - and utility - of combining a strong technological approach with a human-centred / living lab approach.

The rest of the paper is organized as follows. Section 3 first discusses the human-centred / living lab approach and the research objective. Section 4 described the interaction design process: (i) the needs analysis based on Ethnography, which aimed to finely understand work and interaction practices in the retirement home (section 3.2), which informed (ii) the iterative process to design the new multimodal interface (section 3.3). With regards to these insights, future work is discussed in Section 5.

\section{APPROACH AND RESEARCH GOAL}

\subsection{Living lab approach}

The living lab approach combines, from our understanding, [13] a human-centred perspective and a participatory design approach.

\subsubsection{Human-centred}


Human-centred interaction design would be $\mathrm{HCl}$ "centered on the exploration of new forms of living in and through technologies that give primacy to human actors, their values and their activities" [1]. It takes as a starting point human (elderly) capabilities, with a focus on how to support, develop and extend people's capabilities through the latest technological developments [2]. A radically reworked agenda is therefore proposed, for example, on the theme of Ambient Assisted Living. Instead of a technologyfirst or even medical-first approach, Bannon recommends to consider first the fundamental needs and concerns of the ones at the centre of the investigations - the elderly people - so that these AAL technologies could, be both life-saving, but actually add to elderly people's dignity or empowerment.

In order to consider elderlies' needs, Ethnography is used. It offers the opportunity to reveal needs or practices of users which they may not themselves attend to, because they take them so much for granted that they do not think about them, or are too busy [11]. This inability to articulate "needs" is even more true of dependent elderlies.

\subsubsection{Participatory design}

Participatory design (PD) is a cooperative design process, with a focus on enabling different stakeholders with different perspectives and competencies to cooperate. It comprises active user involvement and participation in the design of IT artefacts and systems in professional settings, where it is largely and increasingly used. Designers invite future users to participate in all phases of the design process [5]. PD is generally united by an ethos of empowerment and 'meaningful' involvement for stakeholders in the design of the systems they will use.

Participatory design has traditionally been useful in the design of technology applications or the corealisation of a more holistic socio-technical bricolage of new and existing technologies and practices. Moving away from the traditional computer and "user" notion, e.g. with Ambient Assisted Living technologies [1] or social robots, there is indeed the need for participatory design.

\subsection{Research objective}

The objective of the first step of our research is to design accessible and useful human-robot interaction in a real environment, based on a fine understanding of needs in context. Instead of relying on the existing interface and interaction modalities of the Kompaï 2 robot, we engaged in the project of codesigning and evaluating a new interface and interaction following a living lab approach. However, though this interaction design follows the guidelines of interaction design - whose goal is to create products that enable users to achieve their objective(s) in the best way possible - it is not the end (final aim) of this research. It is the means through which to investigate our primary research object: the acceptance of social robots to improve the quality of life of both residents and personnels of retirement homes. This design process will allow to enquire the research question: is acceptance higher if design follows a human-centred and participatory approach, i.e. if needs/practices have been correctly understood and considered, and if stakeholders have been involved all through the iterative design process?

This research being exploratory and bottom-up, the exact use of the robot is an open question, that will be informed by the insights produced as part of our long-term pilot study (cf. section 5).

\section{DESIGN AND EVALUATION PROCESS}

\subsection{Kompaï 2 in a retirement home}

For this study, the Kompaï 2 robot was used, which we term as "mobile social robot". The fieldwork was done in a retirement home at Troyes, France.

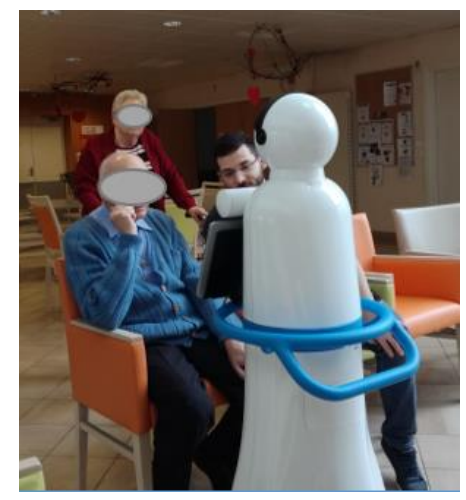

Figure 1: Kompaï tested in the retirement home

The study consisted in (i) Analyzing needs through Ethnography, (ii) Designing interface and interaction informed by these ethnographic insights, (iii) Iterative evaluation and design.

\subsection{Needs analysis informing design}

The Ethnographic fieldwork focused mainly on 4 moments, which emerged as being important in the retirement's home organisation: getting up in the morning, lunch time, animations, transmission meetings and focused on the work nurses, caretakers and assistants in gerontological care. Work activities can be many different tasks ranging from medical care, functional aid, cognitive stimulation - all of which are fundamentally interactional: from simple chatting to talk 
accompanying interaction between bodies. Therefore, communication practices are central to the life of this retirement home. One of the skills and competencies, which emerged from our observations as being essential, is the ability to have efficient interaction with elderlies based on empathy.

After finely observing and analyzing the conversational mechanisms of empathic and benevolent communication, it was possible to extract the "best practices". These are: producing a greeting / summons, allowing time for the resident to understand and respond, informing of all next actions, giving the choice between two possibilities, demonstrating active listening, proposing activities often enough to stimulate without being invasive, repeating without infantilizing, maintaining the balance between friendly and respectful addressing, and always clear verbal formulations. These verbal communication "best practices" have been implemented in the robot's interaction design, mainly the task of proposing activities. The way the questions were designed have been directly informed by the way caretakers trigger interaction by questions, and the time they allow for the elderly to respond.

\subsection{Iterative evaluation and design}

User tests, were done at our University's Living Lab following a protocol specifically designed for our objective : a list of pre-defined tasks, the Wizard of oz technique, complemented with qualitative interviews. They involved 4 users: 3 older adults (independent living retired seniors) and a former caretaker, coherent with Nielsen's [9] recommendations (ideally 3 users and 5 maximum). These user tests allowed to: (i) Confirm the usefulness and necessary simplicity of the functions, (ii) Confirm the appropriateness of the voice interaction, in terms of audibility of text-to-speech, politeness, clarity and effective communication, (iii) Iteratively design a new GUI based on the most appreciated characteristics of the initial 9 interfaces proposed, and users' feedback and suggestions.

A new design was iteratively produced, and served as the basis for new user tests, with 9 residents in the retirement home. There is no room to present in detail the useful feedback these user tests allowed to produce. The main insight is that, despite a general satisfaction about the functions considered as useful, the 9 elderly users were all hindered in their ability to operate tactile interactive devices due to perceptual, cognitive, and motoric changes. Voice modality - both input and output - being much appreciated (except for residents with hearing difficulties), this modality needs to be further examined.

\section{DISCUSSION AND FUTURE WORK}

Together with the interaction modalities, the next step of our research is to enquire the acceptance issue. In particular: is acceptance of a social robot higher following a proper understanding of needs (human-centred approach), and involvement of stakeholders all through the iterative design process (participatory approach)? This research topic will be examined during a long-term pilot evaluation (based on the working prototype described here) in this same retirement home.

This future work aims at contributing to bridging a gap in social robotics studies, both at the level of knowledge production as to methodological reflection. One of the failings pointed by [6] in their review of the studies of social robots, is the methodological shortcomings (short-term, vague). Analyzing social acceptance of social robots in the home context, [7] point to the necessity of long-term studies (to prevent the novelty effect - and because people's perceptions towards robots, their behaviors and their experiences are likely to change over time), in real environments, with a sufficient number of users. This is what the authors intend to do in the next step of this study with this mobile social robot.

\section{CONCLUSION}

This exploratory study has allowed to lay the foundation of a methodologically-sound study of the acceptance of social robotics in retirement homes, which will be the next step of our research. As regards the results from the user tests - which translate a positive perception of this mobile social robot interaction - and the important quantity of valuable knowledge gained from fieldwork, so far, adopting a living lab approach proved helpful in designing a usable, useful and socially acceptable tool. This intermediary "result" will need, as explained supra (section 5), to be examined further. But it seems that this study is on the right path.

\section{ACKNOWLEDGEMENTS}

The authors warmly thank "Les Amis du Living Lab" and the residents and personnels of EHPAD $\mathrm{La}$ Providence of Troyes for their participation in this research.

\section{REFERENCES}

[1] Bannon. L. (2011) Reimagining HCl: Toward a More Human-Centered Perspective. Interactions, 18(4), pages 50-57 
[2] Bannon, Liam (2011) Re-framing $\mathrm{HCl}$ : From human-computer interaction to human-centred interaction design

[3] Bemelmans, R., Gert Jan Gelderblom, Pieter Jonker, Luc de Witte (2012) Socially Assistive Robots in Elderly Care: A Systematic Review into Effects and Effectiveness, Journal of the American Medical Directors Association, Volume 13, Issue 2, Pages 114-120

[4] Bilyea, A., N. Seth, S. Nesathurai, H.A. Abdullah, Robotic assistants in personal care: A scoping review, Medical Engineering \& Physics, Volume 49, Pages 1-6,

[5] Bratteteig, T. and Wagner, I. (2016). Unpacking the Notion of Participation in Participatory Design. Comput. Supported Coop. Work 25, 6, 425-475.

[6] Broekens J., M. Heerink, H. Rosendal (2009) Assistive social robots in elderly care: a review. Gerontechnology; 8(2): 94-103

[7] de Graaf M. M.A., Ben Allouch, S., Klamer, T., (2015) Sharing a life with Harvey: Exploring the acceptance of and relationship-building with a social robot, Computers in Human Behavior, Volume 43, Pages 1-14

[8] Heinzmann J., Zelinsky A. (2000) Building Human-Friendly Robot Systems. In: Hollerbach J.M., Koditschek D.E. (eds) Robotics Research. Springer, London

[9] Nielsen, J., (1994) Usability engineering, Morgan Kaufmann Publishers Inc. San Francisco, CA

[10] Pineau, J., Montemerlo, M., Pollack, M., Roy, N., Thrun S., (2003) Towards robotic assistants in nursing homes: Challenges and results, Robotics and Autonomous Systems 42, pages 271-281

[11] Randall, D., (2007) Richard Harper, Mark Rouncefield Fieldwork for Design: Theory and Practice April 2007

[12] Shishehgar, M., Donald Kerr, Jacqueline Blake (2018, in press) A systematic review of research into how robotic technology can help older people, Smart Health

[13] Voilmy, D., (2017) Les « living labs » et la conception participative : l'exemple d'ActivAgeing, numéro spécial : Les gérontechnologies, mythe ou réalité ?, Retraite et Société, $n^{\circ} 75$, pages $125-136$

[14] Zinn, M., Bernard Roth, Oussama Khatib, J. Kenneth Salisbury (2004) A New Actuation Approach for Human Friendly Robot Design. The International Journal of Robotics Research, Vol 23, Issue 4-5, pp. 379 - 398 